\title{
Best of Breed Control of Platinum Precipitation Reactors
}

\author{
Rotimi Agbebi ${ }^{\mathrm{a}}$, Carl Sandrock ${ }^{\mathrm{b}}$ \\ ${ }^{a}$ University of Pretoria, 1 Lynnwood Road, Pretoria 002, South Africa \\ ${ }^{b}$ University of Pretoria, 1 Lynnwood Road, Pretoria 002, South Africa \\ carl.sandrock@up.ac.za
}

\begin{abstract}
An existing batch precipitation reactor in the mining industry was modelled from first principles using MATLAB S-function language and wrapped into Simulink custom blocks. The model was validated with open loop simulations to evaluate the response to step changes in the model inputs. Two PID controllers with different parameters were implemented on the model at different operating points and their temperature control performance was evaluated based on their ability track a temperature of the industrial reactor as a set-point. The performance of the controllers was measured and compared using an integral time-domain performance measure. A commercial advanced process controller (APC) was implemented on the model, a communication interface between the model in Simulink and the commercial controller was developed with Industrial Data Xchange (IDX); an OPC client and server.
\end{abstract}

Keywords: Batch Reactors, PID, APC, Aspen DMC plus, OPC

\section{Introduction}

Batch reactors are integral to the refining process of platinum group metals and their effective control is essential to ensure safe and efficient process in minerals industry (Singh et al. 2010). In these reactors various process phases are encountered in a single process batch; they are heating, cooling and reaction phase. These reactions occur as either an exothermic or endothermic reaction and the control of the batch reactors is essentially treated as a temperature control problem (Friedrich and Perne, 1996). A change from one batch to another batch could necessitate a change in control actions and this makes the control more difficult as different models are applicable for different batch process recipes operating at different operating conditions (Singh et al. 2010).

In many industrial applications, the temperature control of the reactor is carried out with PID controllers in a cascade structure, however rapid and precise temperature control is hard to achieve with these controllers (Stampar et al. 2013).

In terms of a PID controller, the two actuators on the steam and cooling inlet valve to the jacket can be considered as one manipulated variable and a split-range algorithm can be implemented as part of the controlled system (Balaton et al. 2012). Using one controller as long as the system state is close to the corresponding operating point and switching to another when the operating point is sufficiently close to the next operating point gives a better controller performance (Rugh and Shamma, 2000). The use of advanced process control (APC) systems has the potential to improve the control of batch reactors employed in the refining of PGMs (platinum group metals) (Singh et al. 2010). Most of the literature covers process control of continuous reactors in petrochemical industries, reactors with individual heating and cooling jackets. A few literatures cover the application of advanced process control on batch reactors in the minerals industry (Singh et al. 2010). Our aim is to build a platform on which the 
performance of the various controllers can be compared and an algorithm to switch between the various controllers for particular process conditions.

\section{Process Model Description}

The first phase in the reactor involves heating, inflow of steam to achieve a desired temperature set-point. The reaction phase starts at the desired temperature where reagents are added, this result to an exothermic reaction which requires cooling, and then the cooling phase starts with inflow of cooling water (Singh et al. 2010). The temperature control performance mainly depends on the heating-cooling system associated with the reactor. The different configurations of heating-cooling systems cited in literature can be classified into two types: multifluid and monofluid systems (Louleh et al.1999). The commercial reactor modeled in this study has a multifluid system where steam is used as heating medium and water used as a cooling medium as shown in the figure 1 below. The transfer of heat energy to and from the jacket and the reactor is achieved with the transfer fluid through the wall of the reactor. Two exothermic chemical reactions were modelled in the reactor;

Dissolution of Ruthenium

$$
R u_{s}+3 C l_{2(g)} \rightarrow R u C l_{3(l)}
$$

Evaporation of Ruthenium Chloride

$$
\begin{aligned}
2 \mathrm{H}_{2} \mathrm{O}_{(\mathrm{l})}+2 \mathrm{RuCl}_{3(\mathrm{l})}+2 \mathrm{NaClO}_{3(\mathrm{l})} & \rightarrow \\
4 \mathrm{HCl}_{(\mathrm{l})} & +2 \mathrm{RuO}_{4(\mathrm{~g})}+\mathrm{Cl}_{2(\mathrm{~g})}+ \\
2 \mathrm{NaCl}_{(\mathrm{l})} & (2)
\end{aligned}
$$

The model was developed in an explicit formulation using MATLAB s-function which could be used directly in custom blocks in SIMULINK in pursuit of getting the reactor model to interact effectively with external platform i.e. commercial controllers

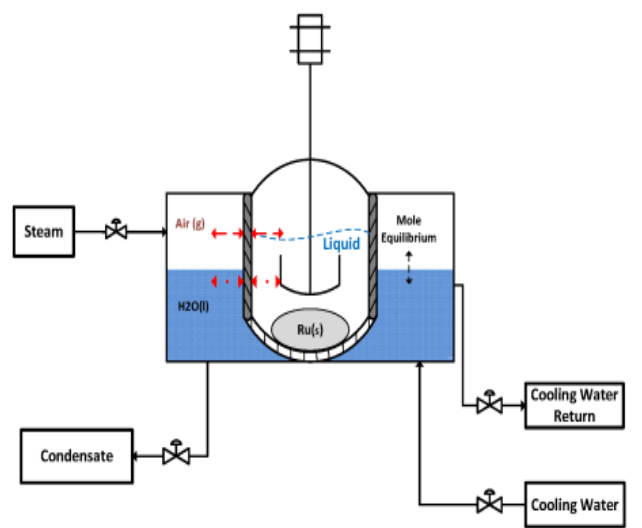

Figure 1: Reactor Model Description

\section{PID Control}

The process operating conditions are different from phase to phase and also from batch to batch. As such, a requirement on the controller is that it regulates the plant at each operating point or regime. The reactor temperature control is dependent on the control of the steam valve and the cooling water valve of a reactor with a single jacket.

For this design requirement to be correctly modeled using a PID Controller, a splitrange algorithm was implemented. The split-range algorithm is preferred in the control of cascaded reactor, to operate two actuators which control the inflow of utilities at the different times during the different phases of a single batch (Balaton et al. 2012).

The two actuators on the steam and cooling water inflow valve to the jacket were considered as one manipulated variable (MV) and the temperature of the reactor as the controlled variable (CV). The PID controllers perform better on operating points they were tuned on. The performance of PIDs degrades with change in process regimes and operating points (Singh et al. 2010). Two PID controllers with different controller 
parameters and set points were implemented on the SIMULINK model at different model operating points.

\section{Control Objective and Performance Measure}

The controller design objective was its set-point tracking ability; its performance can be measured with several integral time-domain performance measures. They are Integral of the Square of the Error (ISE), the Integral of the Absolute value of the Error (IAE), and the Integral of the time-weighted Absolute Error (ITAE).

$$
E=\int_{0}^{\infty} f(t) \delta t
$$

Where $f(t)$ is a function of $\epsilon(t)=y_{s p}(t)-y(t)$

These were all implemented in the performance block of the Simulink model; however the Integral of the Square of the Error (ISE) was used to evaluate the performance of the controllers.

\section{Advanced Process Control.}

A commercial MPC controller called DMCplus; which is a multivariable controller from AspenTech was developed to control the temperature of the reactor model in Simulink. The DMCplus controller implemented in this work has two manipulated variables (steam and cooling water valves to the same reactor jacket), no feed forward variables and one controlled variable (reactor temperature). As both manipulated variables use the same jacket, they cannot be used at the same time. The controller executes periodically in cycles, the following steps were followed in developing the controller;

- Configuration of MVs and CV tags using Aspen DMC plus Build

- Calculate the open loop prediction for the controlled variable based on step change in manipulated variables which is the model development

- Steady State Simulation using Aspen DMC plus Model

- Determine the path by which the manipulated variables move from their current positions to the end positions i.e. the move plan of the controller.

- Prediction and Tuning of Controller using ASPEN APC web Interface

\subsection{Model Interaction with external platforms.}

The communication between the Simulink model and the commercial controller shown in figure 2 is very important and this was achieved using MATLAB OPC toolbox and Industrial Data Xchange (IDX); an OPC client and server. The IDX makes the outputs and inputs of the Simulink model available to the ASPEN DMC plus as data tags through the Aspen Cim-IO.The Aspen Cim-IO is an interface solution for AspenTech systems.

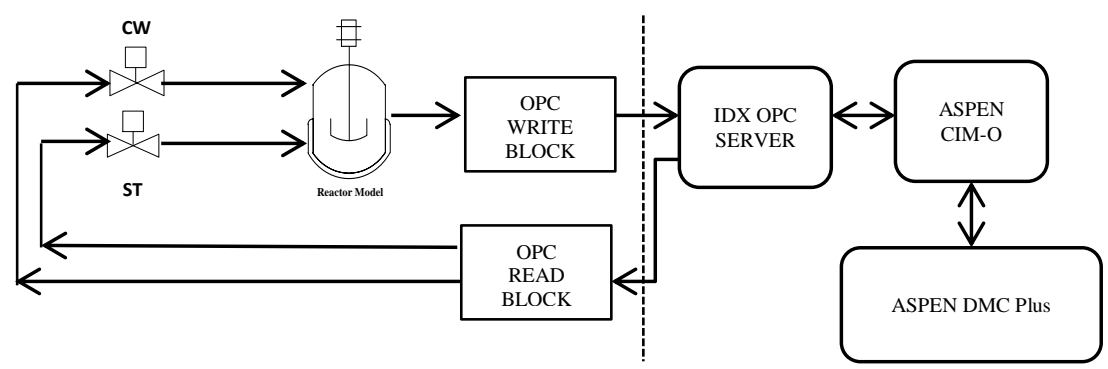

Figure 2 : Simulink model interaction with commercial controller 
F_. IDX_PMR.1 - IDXOnline OPC Client (Beta)

File $\mathrm{OPC}$ View

\begin{tabular}{|l|l|l|}
\hline Tag & Value & Time \\
\hline CWwrvdep & 1 & $11 / 26 / 1415: 28: 37$ \\
FhxH20gsp & 0 & $11 / 26 / 1413: 11: 40$ \\
FhxH20gi & 0 & $11 / 26 / 1415: 27: 27$ \\
FhxH20li & 84 & $11 / 26 / 1415: 48: 10$ \\
FhxH20lsp & 91 & $11 / 26 / 1415: 48: 12$ \\
stsrvdep & 0 & $11 / 26 / 1415: 27: 42$ \\
trxl & 303.644 & $11 / 26 / 1415: 48: 10$ \\
trxsp & 299.208 & $11 / 26 / 1415: 48: 10$
\end{tabular}

Figure 3 shows the IDX OPC client interface with the MVs and CVs data communication between the Simulink model and commercial controller.

Figure 3: IDX OPC Client

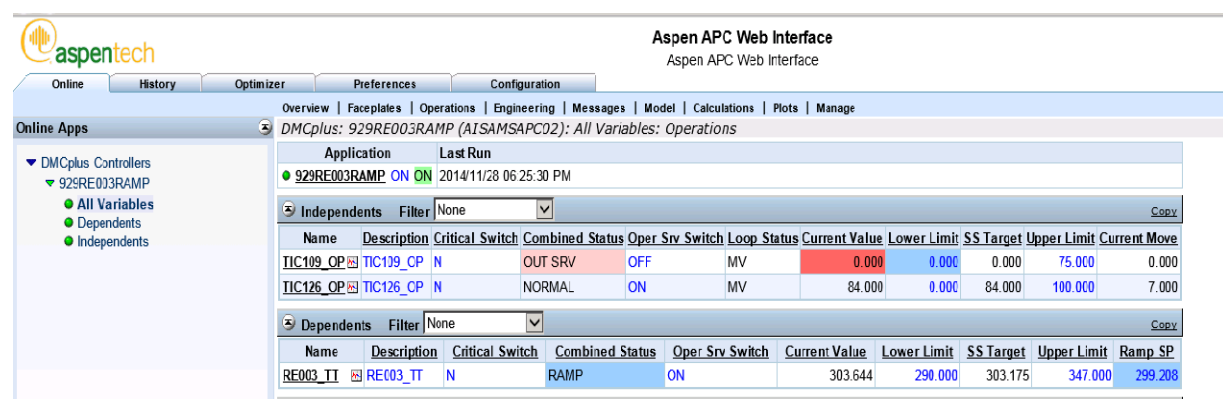

Figure 4: ASPEN APC Web Interface

Figure 4 shows the independent and dependent variables of the controller configuration developed using Aspen DMC plus Build, and monitored on the APC Web Interface. A controller model was built using the Aspen DMC plus Model, the open loop prediction of the model is shown in figure 5. This shows a test controller with cooling phase; increase in cooling water and decrease in steam.

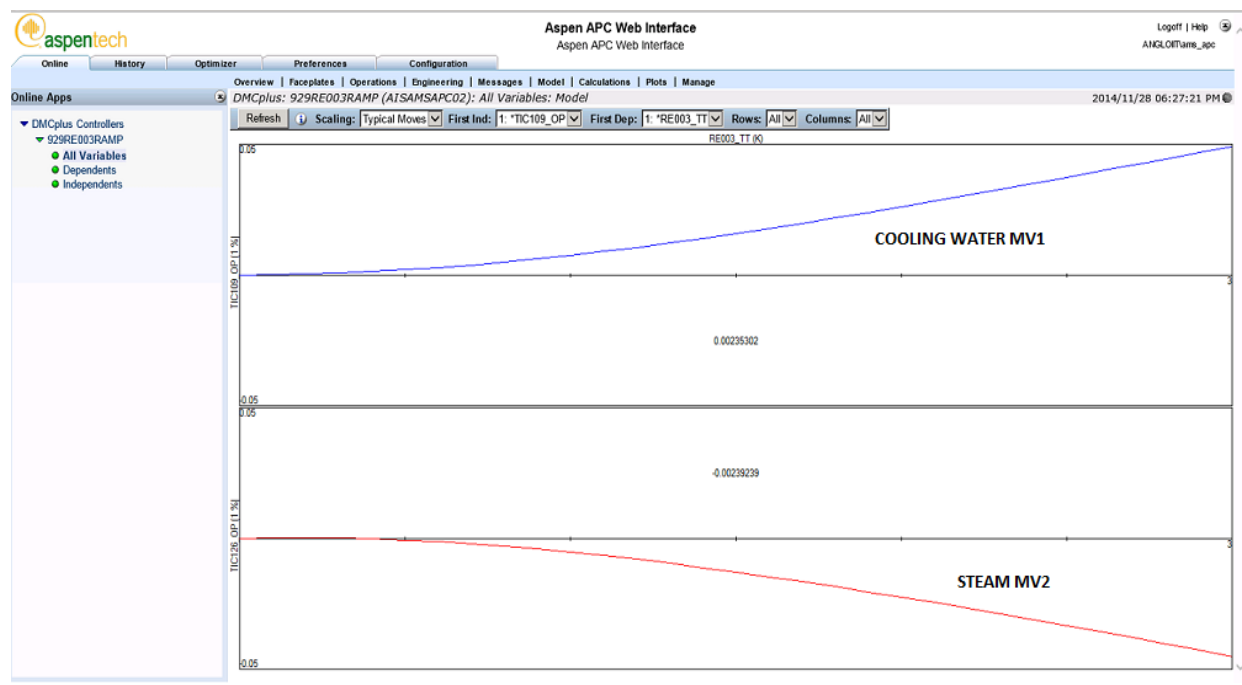

Figure 5: Open Loop Simulation.

Figure 6 shows a closed loop test simulation of the model, the response of the CV to predicted moves of the MVs; this validates the communication between the variables. 
Best of breed control of platinum precipitation reactors

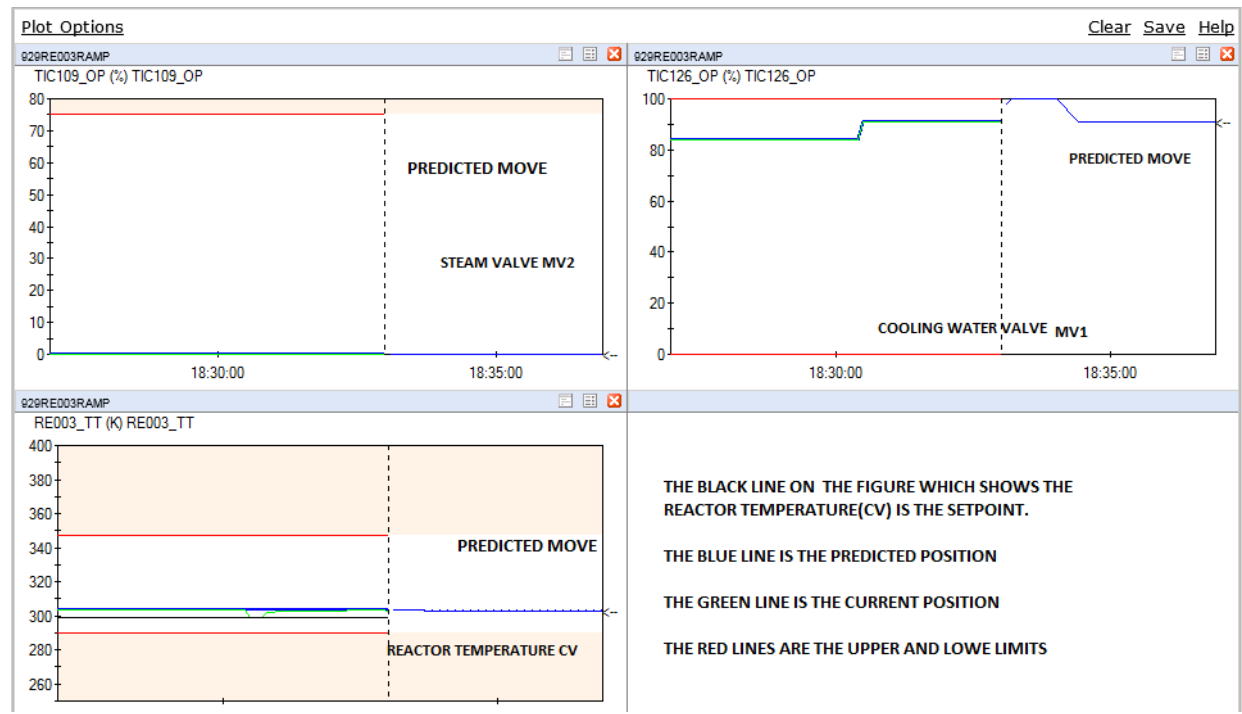

Figure 6: Closed Loop Simulation Response

\section{Results and Discussion}

The Simulink model was linearized to obtain linear time-invariant state-space models at two different steady state operating points, open loop simulations were ran on two the models and closed loop models were developed using Simulink PID compensator design tool.

The open loop response of the reactor temperature to a step change in the inflow of utilities; steam inflow for heating and cooling water inflow for cooling was done. This validates the response of the model to a unit step change in input at set utility temperatures which are cooling water at $278 \mathrm{~K}$ and steam at $400.15 \mathrm{~K}$.

The closed loop performance results of the PID controllers show the model reactor tracking the set point temperature which is the plant temperature data.
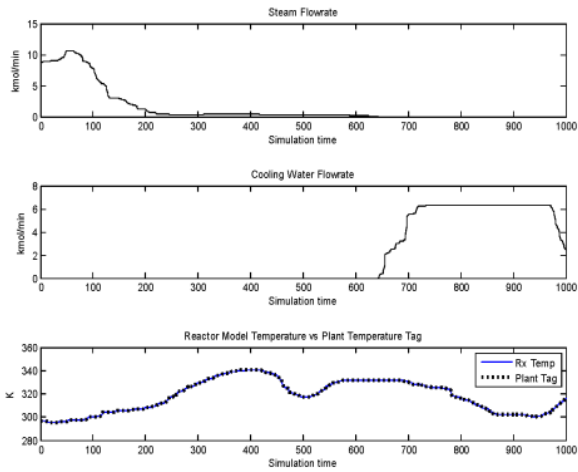

Figure 10: Simulation results of PID 1 on model 1
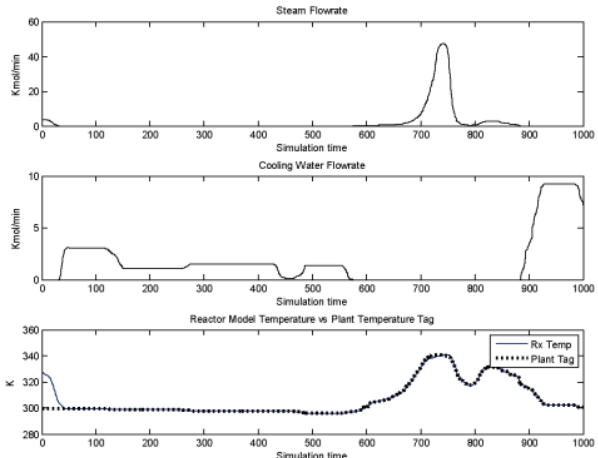

Figure 11: Simulation with PID 2 on model 1

Figure 10 and Figure 11 shows closed loop simulation using using temperature from plant data as PID set point. In Figure 10, PID controller 1 tuned on model 1 shows a very good set-point tracking performance with a lower integral square error of 5.995. 
In Figure 11, PID controller 2 tuned on model 1 shows a bad set-point tracking performance with a higher integral square error of 132.62 .

Table 1 shows different operating point of the linearized state-space models and Table 2 shows the performance of different controllers implemented on different models using temperature from plant data as reference temperature. The PID 1 performed better on model 1 and PID 2 performed better on model 2

Table 1: Model operating points

Table 2: Controller Performance Values

\begin{tabular}{|c|c|c|c|c|c|}
\hline Operating Points & Model 1 & $\begin{array}{c}\text { Model } \\
2\end{array}$ & Controller & Model & $\begin{array}{c}\text { Integral Square } \\
\text { of Error (ISE) }\end{array}$ \\
\hline \multirow{2}{*}{$\begin{array}{c}\text { Reactor } \\
\text { Temperature (K) }\end{array}$} & \multirow{2}{*}{303.1040} & \multirow[b]{2}{*}{292.1161} & PID 1 & 1 & 5.995 \\
\hline & & & PID 1 & 2 & 87.61 \\
\hline \multirow{2}{*}{$\begin{array}{c}\text { Jacket } \\
\text { Temperature (K) }\end{array}$} & \multirow{2}{*}{304.1142} & \multirow{2}{*}{299.0432} & PID 2 & 1 & 132.62 \\
\hline & & & PID 2 & 2 & 7.993 \\
\hline
\end{tabular}

\section{Further Work}

The Advanced Process Controller (APC) implemented on the reactor model will be tuned to its best performance and the performance will be compared to that of the PID controllers and a combination of controllers will be implemented on the model in order to assess if the hybrid controller will out-perform a single controller.

\section{Conclusions}

PID controllers perform better on model operating points there were tuned on with a lower integral square error (ISE) and their performance degrades with change in process operating points and batches (Noguchi and Kobari, 2005). To overcome these limitations, a commercial APC (Aspen DMC Plus) which has been successfully connected to the Simulink model will be tuned and completely implemented to validate (Singh et al. 2010)'s work on the actual commercial reactors which proves that the APC controller out-performs PID controller. The platform needed to compare ability of the PID and the APC control system with the reactor model is now developed.

\section{References}

M.G. Balaton, L. Nagy, F. Szeifert, 2012, Model-Based Split-Range Algorithm for the Temperature Control of a Batch Reactor. Scientific Research Eng, 4, 515-525

M. Friedrich and R. Perne, 1995, Design and Control of Batch Reactors - An Industrial Viewpoint, Comp \& Chem Eng, 19, S357-S368.

Z. Louleh, M. Cabassud, M.V. Le Lann, 1999, A New Strategy for Temperature Control of Batch Reactors: Experimental Application, Chem Eng Jour 75, 1, 11-20.

Y. Noguchi and M. Kobari, 2005, Model Predictive Control with single heat transfer fluid for Batch Reactor temperature control, SICE Annual Conference, 449 - 452

W. J. Rugh and J. S. Shamma, 2000, Research on gain scheduling, Automatica, 36, $1401-1425$

A. Singh, P.G.R. De Villiers, P. Rambalee, G. Gous, J. De Klerk, Humphries G. , 2010, A Holistic approach to the application of Model Predictive Control to batch reactor, Automation in Mining, Mineral and Metal Processing, 13, 172-132

S. Stampar and S. Somkolic, 2013, Nonlinear Control of Hybrid Batch Reactor, Journal of Mech Eng , 59, $112-123$ 